\title{
Acceleration of Microwave-Assisted Enzymatic Digestion Reactions by Magnetite Beads
}

\author{
Wei-Yu Chen ${ }^{1}$ and Yu-Chie Chen ${ }^{* 1,2}$ \\ Department of Applied Chemistry ${ }^{1}$ and Institute of Molecular Science ${ }^{2}$ \\ National Chiao Tung University, Hsinchu 300, Taiwan
}

\section{Supporting Information}

Figure 1 presents the TEM image of the magnetic beads, which have a particle size of $\sim 20 \mathrm{~nm}$.

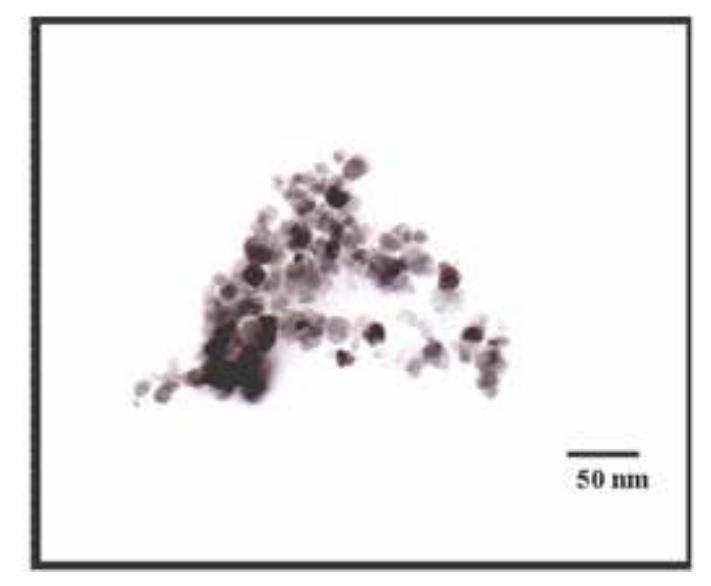

Figure 1. TEM image of the magnetite beads

Figures 2a-c present the MALDI mass spectra of the tryptic digest product of cytochrome $\mathrm{C}$ obtained under microwave heating for $30 \mathrm{sec}$ in different solvent systems, without adding magnetite beads as the irradiation absorbers. The ions derived from $\mathrm{M}^{+}, \mathrm{M}^{2+}$, and $\mathrm{M}^{3+}$ of cytochrome $\mathrm{C}$ appear in the mass spectra. No noticeable hydrolysis has occurred in this short period of microwave-heating. 


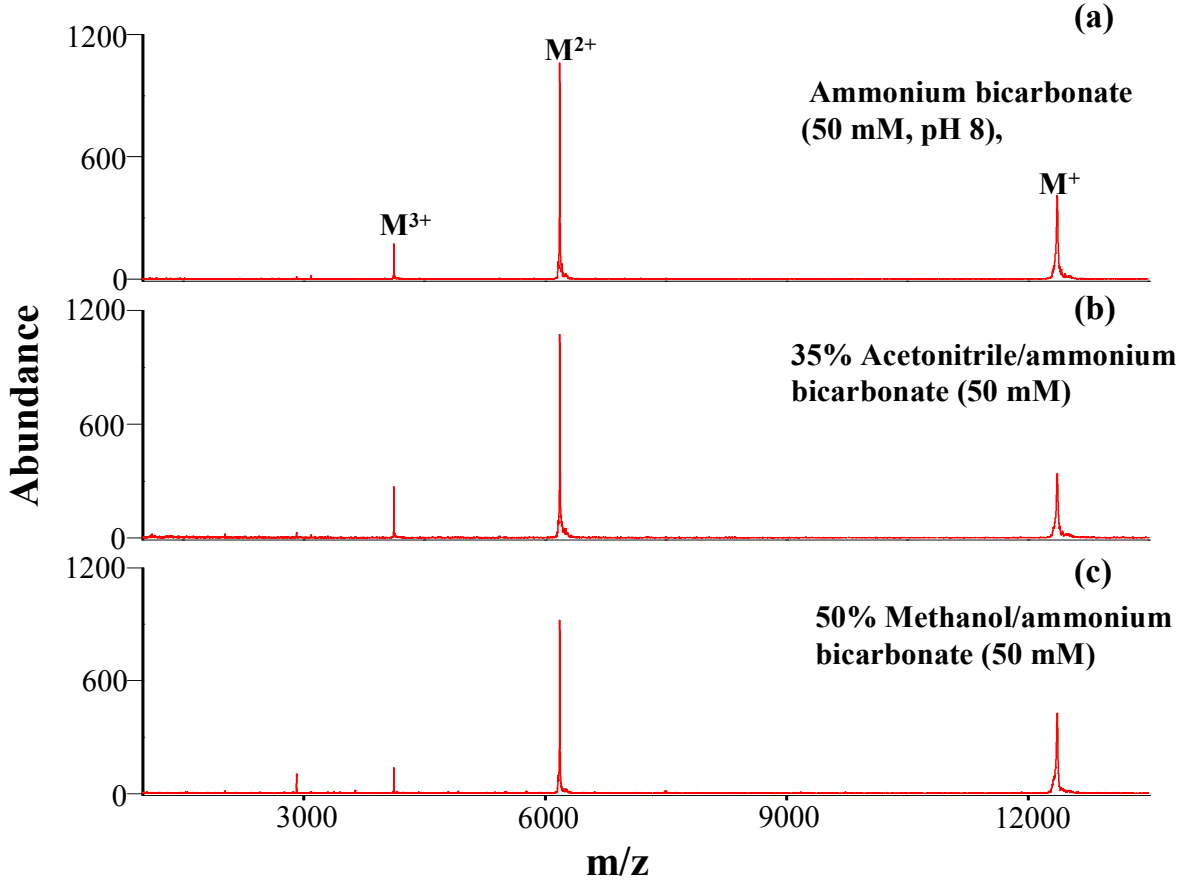

Figure 2. MALDI mass spectra of the tryptic digest product of cytochrome $\mathrm{C}$ prepared in (a) ammonium bicarbonate aqueous solution (50 mM, pH 8), (b) $35 \%$ acetonitrile/ammonium bicarbonate solution $(50 \mathrm{mM})$, and (c) $50 \%$

methanol/ammonium bicarbonate $(50 \mathrm{mM})$ obtained under microwave heating for 30 sec.
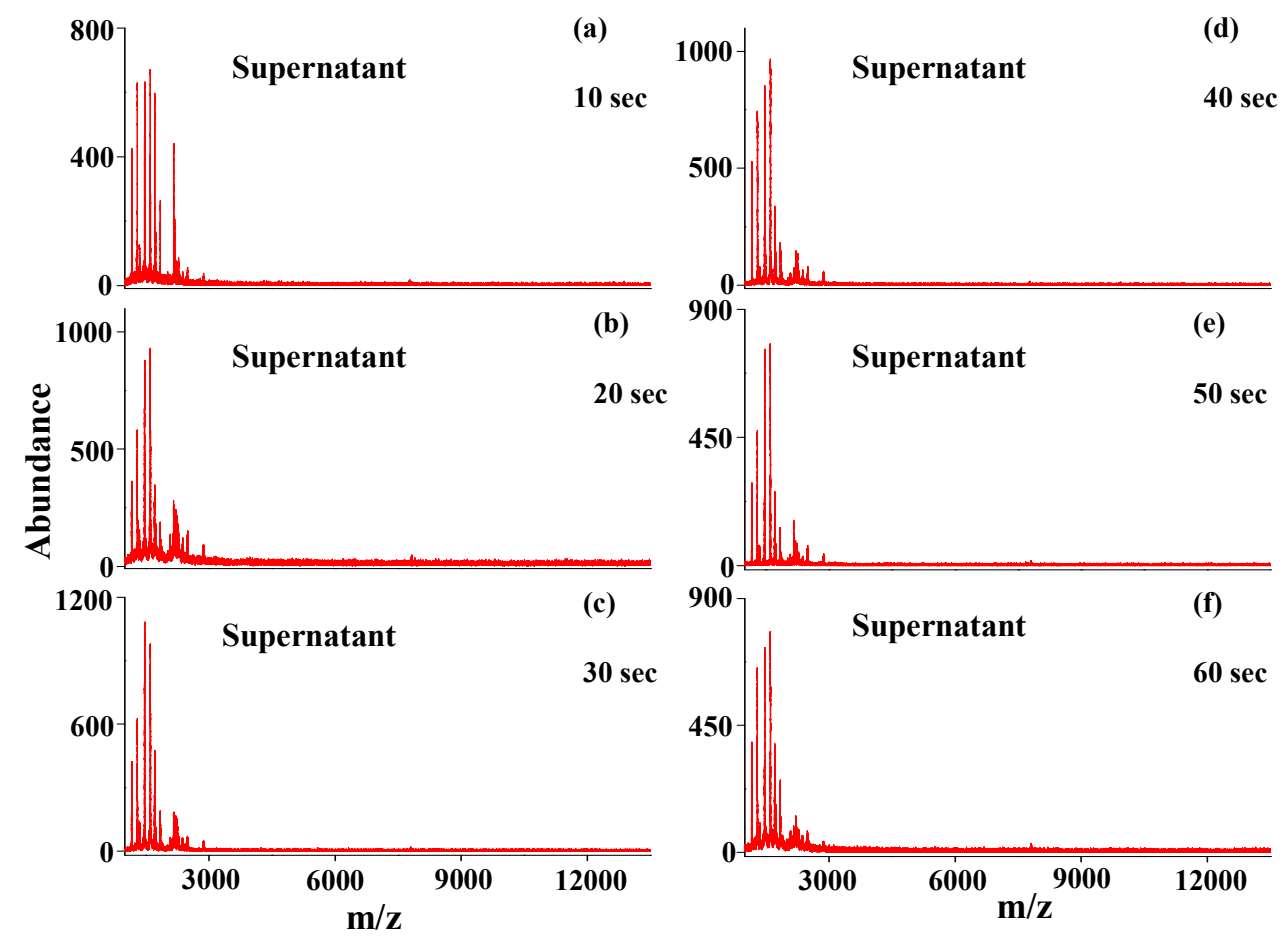

Figure 3. MALDI mass spectra of the supernatant obtained from the tryptic digest of cytochrome $\mathrm{C}\left(10^{-5} \mathrm{M}, 50 \mu \mathrm{l}\right)$ in ammonium bicarbonate $(50 \mathrm{mM}, \mathrm{pH} 8)$ with the addition of $600 \mu \mathrm{g}$ of the magnetic beads, under microwave heating for (a) $10 \mathrm{sec}$, (b) $20 \mathrm{sec}$, (c) $30 \mathrm{sec}$, (d) $40 \mathrm{sec}$, (e) $50 \mathrm{sec}$, (f) $60 \mathrm{sec}$. 


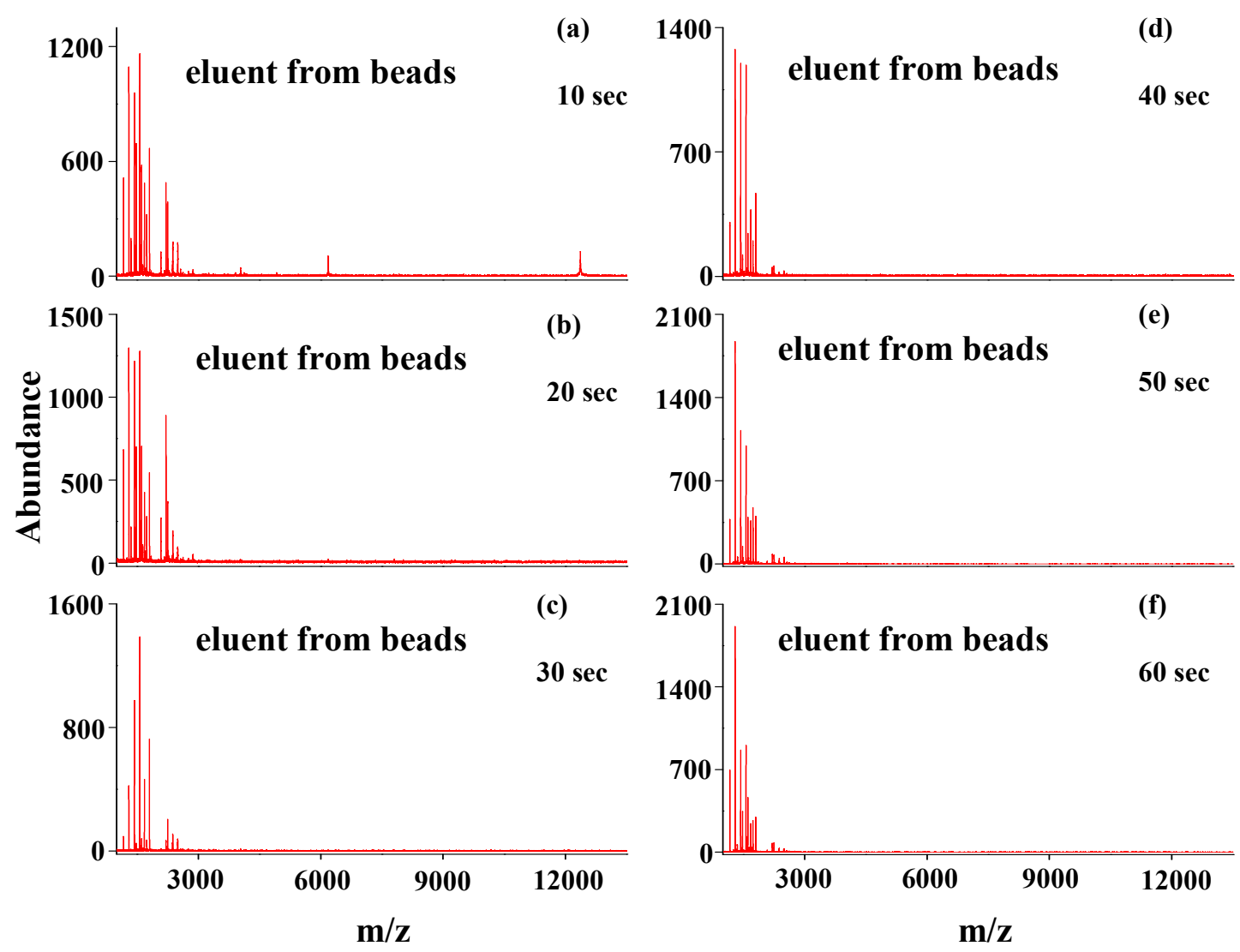

Figure 4. MALDI mass spectra of the sample eluted from the beads obtained from the tryptic digest of cytochrome $\mathrm{C}\left(10^{-5} \mathrm{M}, 50 \mu \mathrm{l}\right)$ prepared in ammonium bicarbonate $(50$ $\mathrm{mM}, \mathrm{pH} 8$ ) with the addition of $600 \mu \mathrm{g}$ of the magnetic beads, under microwave heating for (a) $10 \mathrm{sec}$, (b) $20 \mathrm{sec}$, (c) $30 \mathrm{sec}$, (d) $40 \mathrm{sec}$, (e) $50 \mathrm{sec}$, (f) $60 \mathrm{sec}$.

Table 1. Peptide ion peaks observed in Figure 5c

$\begin{array}{cccl}\begin{array}{c}\text { Observed } \\ {[\mathrm{M}+\mathrm{H}]^{+}}\end{array} & \begin{array}{c}\text { Calculated } \\ {[\mathrm{M}+\mathrm{H}]^{+}}\end{array} & \begin{array}{c}\text { Missed } \\ \text { Cleavage }\end{array} & \text { Peptide Sequence } \\ \mathbf{1 3 6 0 . 7 6} & 1360.75 & 1 & \text { ALELFRNDIAAK (134-145) } \\ 1936.62 & 1937.01 & 2 & \text { LFTGHPETLEKFDKFK (32-47) } \\ 2282.91 & 2283.21 & 3 & \text { ALELFRNDIAAKYKELGFQG (134-153) } \\ 3004.39 & 3004.56 & 4 & \text { LFTGHPETLEKFDKFKHLKTEAEMK (32-56) } \\ 3403.75 & 3403.73 & 1 & \text { GLSDGEWQQVLNVWGKVEADIAGHGQEVLIR (1-31) } \\ 3775.93 & 3775.97 & 6 & \text { LFTGHPETLEKFDKFKHLKTEAEMKASEDLKK (32- }\end{array}$


Table 2. Peptide ion peaks observed in Figure 5g

$\begin{array}{cccl}\begin{array}{c}\text { Observed } \\ {[\mathrm{M}+\mathrm{H}]^{+}}\end{array} & \begin{array}{c}\text { Calculated } \\ {[\mathrm{M}+\mathrm{H}]^{+}}\end{array} & \begin{array}{c}\text { Missed } \\ \text { Cleavage }\end{array} & \begin{array}{l}\text { Peptide Sequence } \\ 1360.75\end{array} \\ 1360.75 & 1 & \text { ALELFRNDIAAK (134-145) } \\ 1378.67 & 1378.84 & 0 & \text { HGTVVLTALGGILK (64-77) } \\ 1506.64 & 1506.93 & 1 & \text { KHGTVVLTALGGILK (63-77) } \\ 1506.64 & 1506.93 & 1 & \text { HGTVVLTALGGILKK (64-78) } \\ 1634.65 & 1635.03 & 2 & \text { KHGTVVLTALGGILKK (63-78) } \\ 1634.65 & 1635.03 & 2 & \text { HGTVVLTALGGILKKK (64-79) } \\ 1651.91 & 1651.91 & 2 & \text { ALELFRNDIAAKYK (134-147) } \\ 1853.58 & 1853.96 & 0 & \text { GHHEAELKPLAQSHATK (80-96) } \\ 1857.52 & 1857.97 & 3 & \text { HLKTEAEMKASEDLKK (48-63) } \\ 1936.65 & 1937.01 & 2 & \text { LFTGHPETLEKFDKFK (32-47) } \\ 1981.67 & 1982.05 & 1 & \text { KGHHEAELKPLAQSHATK (79-96) } \\ 2109.70 & 2110.15 & 2 & \text { KKGHHEAELKPLAQSHATK (78-96) } \\ 2282.84 & 2283.21 & 3 & \text { ALELFRNDIAAKYKELGFQG (134-153) } \\ 2336.33 & 2336.33 & 1 & \text { IPIKYLEFISDAIIHVLHSK (99-118) } \\ 2698.52 & 2698.52 & 3 & \text { KGHHEAELKPLAQSHATKHKIPIK (79-102) } \\ 2826.62 & 2826.62 & 4 & \text { KKGHHEAELKPLAQSHATKHKIPIK (78-102) } \\ 3004.56 & 3004.56 & 4 & \text { LFTGHPETLEKFDKFKHLKTEAEMK (32-56) } \\ 3403.63 & 3403.73 & 1 & \text { GLSDGEWQQVLNVWGKVEADIAGHGQEVLIR (1-31) } \\ 3469.97 & 3469.97 & 3 & \text { HGTVVLTALGGILKKKGHHEAELKPLAQSHATK (64- } \\ & & & \text { 96) }\end{array}$

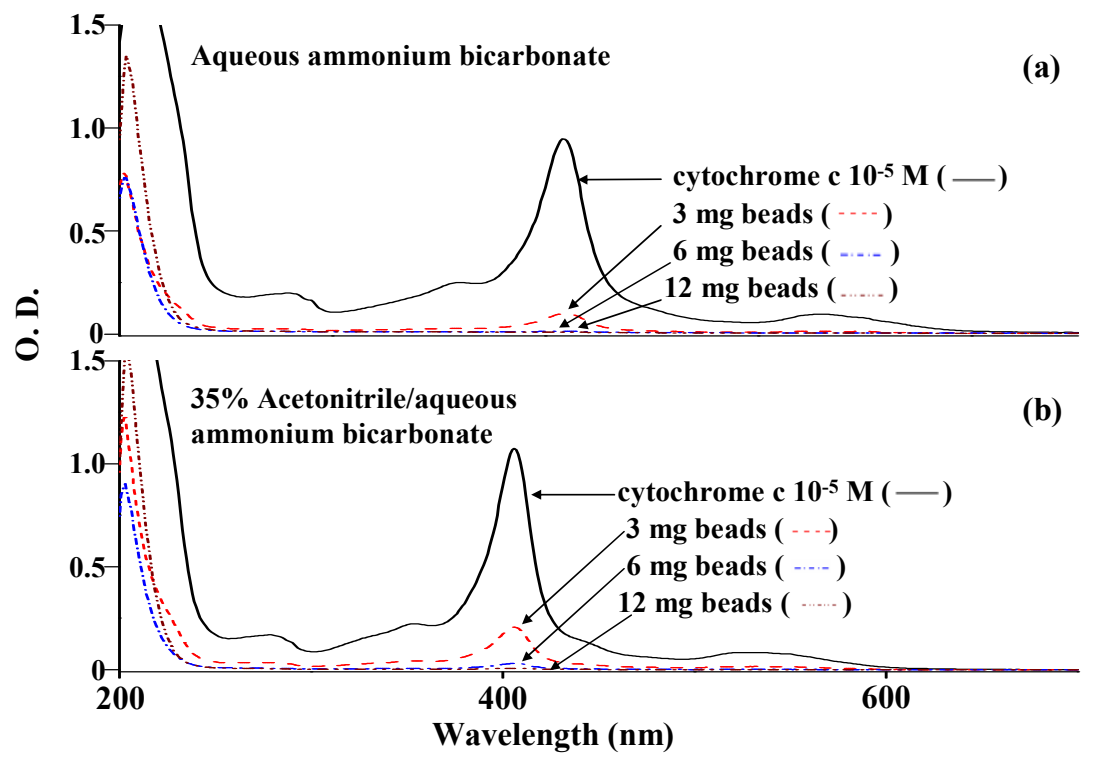

Figure 5. Absorption spectra of the supernatant obtained using various amounts of magnetic beads ( $3 \mathrm{mg}, 6 \mathrm{mg}$, and $12 \mathrm{mg}$ ) to trap target species from the sample containing cytochrome $\mathrm{C}\left(1 \mathrm{~mL}, 10^{-5} \mathrm{M}\right)$ in (a) ammonium bicarbonate aqueous solution $(50 \mathrm{mM}, \mathrm{pH} 8)$ and (b) $35 \%$ acetonitrile/ammonium bicarbonate solution (50 $\mathrm{mM})$. 


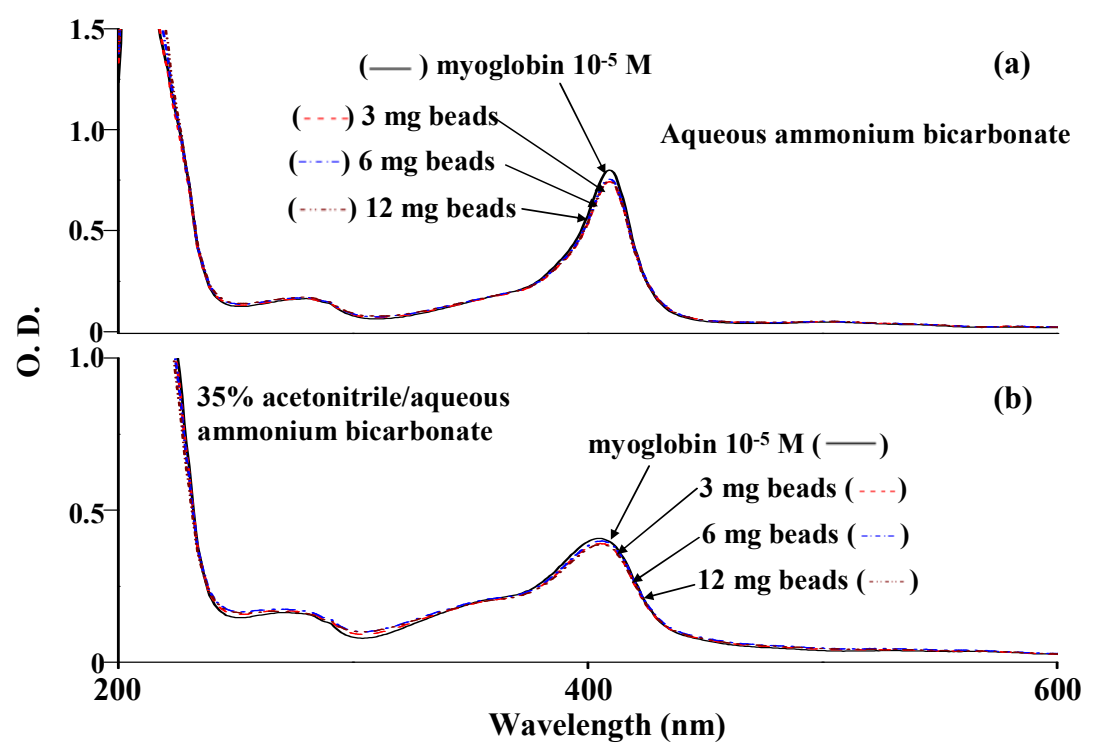

Figure 6. Absorption spectra of the supernatant obtained using various amounts of magnetic beads ( $3 \mathrm{mg}, 6 \mathrm{mg}$, and $12 \mathrm{mg}$ ) to trap target species from the sample containing myoglobin $\left(1 \mathrm{~mL}, 10^{-5} \mathrm{M}\right)$ in (a) aqueous ammonium bicarbonate $(50 \mathrm{mM}$, $\mathrm{pH} 8$ ) and (b) $35 \%$ acetonitrile/ammonium bicarbonate solution (50 mM).

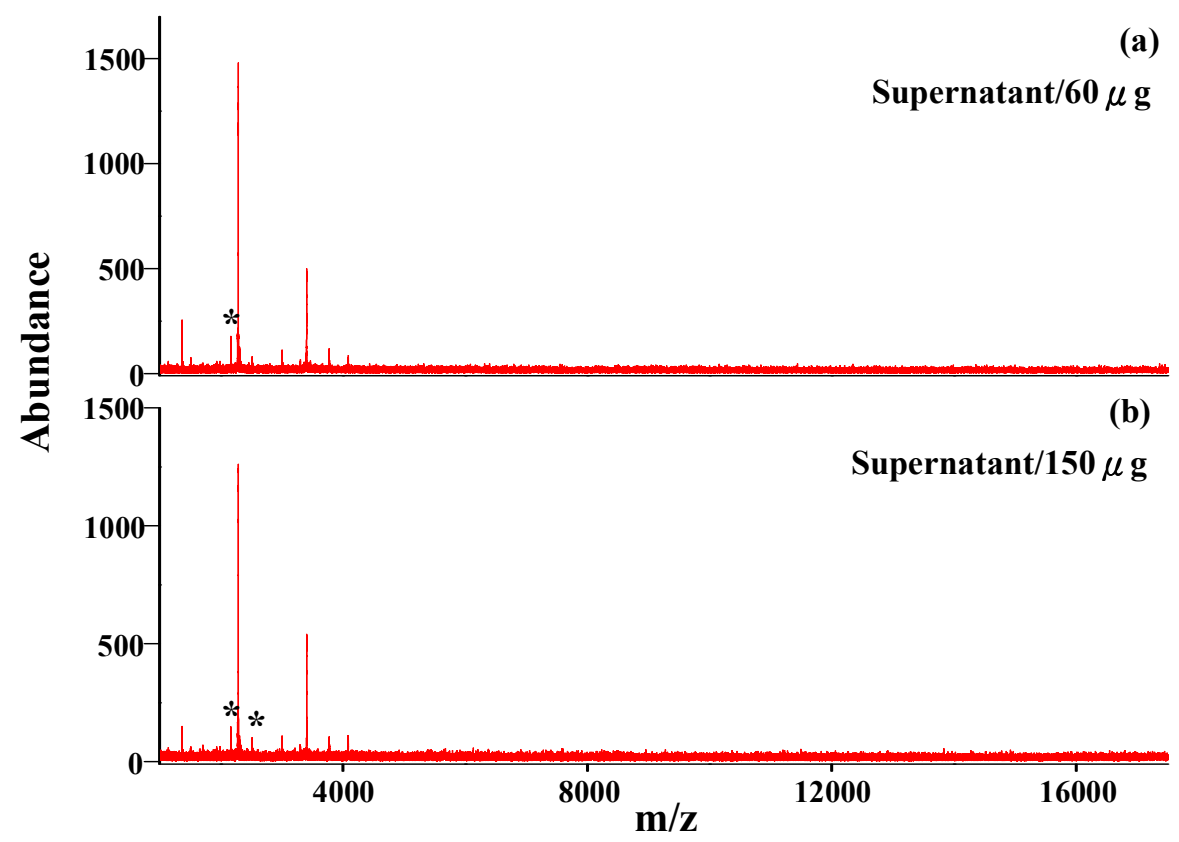

Figure 7. MALDI mass spectra of the supernatant obtained from the tryptic digest of myoglobin $\left(10^{-5} \mathrm{M}, 50 \mu \mathrm{l}\right)$ in $35 \%$ acetonitrile/ammonium bicarbonate $(50 \mathrm{mM})$ with (a) $60 \mu \mathrm{g}$, (b) $150 \mu \mathrm{g}$ of trypsin immobilized magnetic beads, under microwave heating for $1 \mathrm{~min}$. The peaks marked with asterisks were the peptides derived from trypsin autolysis. 